\title{
MS39-01 | Ultrafast Dynamics of Chemistry in Solution Studied by Time-Resolved X-RAy Diffuse Scattering at LCLS
}

van Driel, Tim (LCLS / SLAC National Accelerator Laboratory, Menlo Park, USA)

Laser pump / X-ray probe experiments provide novel fundamental insight into ultrafast photo-induced processes. The arrival of X-ray Free Electron Lasers has pushed the time-resolution of such experiments to sub $100 \mathrm{fs}$.

Here I will present the current capabilities of the XPP [1] and XCS [2] instruments at LCLS for the purpose of studying solution phase chemistry. This will include instrumentation, diagnostics and performance as well as some published and unpublished scientific/technical examples [3]. Furthermore I will describe and elaborate on the future upgrades of LCLS-II and LCLS-II-HE and the potential improvements we hope to achieve.

Recently improvements include implementing the setup at the XCS endstation, a new liquid sample chamber, new liquid delivery nozzles as well as a newly developed epix10k high dynamic range detector for scattering. These technical improvements in combination with improved analysis, diagnostics and real-time feedback have greatly improved the fidelity of such experiments in addition to decreasing the time spent on alignment and setup.

This makes it possible to accurately measure the time-resolved diffuse scattering to follow the photo-induced dynamics of molecules in solution. Combined with X-ray spectroscopic techniques, such measurements can provide a novel insight into excited state dynamics, solvent interactions as well as provide a direct measurement of the structure, spin and charge of many photo active molecules on the intrinsic timescale of such ultra-fast molecular processes.

[1] M.Chollet et al., J. Synchrotron Rad. 22, 503-507. (2015)

[2] R.Alonso-Mori et al.,J. Synchrotron Rad. 22, 508-513. (2015)

[3] van Driel et al., Nat. Comm. 7, 13678 (2016) 